\title{
SOBRE LA PRODUCCIÓN Y FORMACIÓN DE BENITO, PINTOR DEL RENACIMIENTO PALENTINO *
}

\author{
POR \\ MATTHIAS WENIGER \\ Staatliche Museen zu Berlin
}

\begin{abstract}
Up to this point just a few isolated paintings have been connected with Benito, an artist of Palencia known only by his first name. This first monographic study attempts to enrich his catalogue with several new works, most important among them his contribution to the High Altarpiece of St. Peter's Church in San Pedro de Montealegre (today in the province of Valladolid, but once part of the diocesis of Palencia). The existence of several paintings close to his manner but not necessarily due to Benito himself proves that his art had in its day not been an isolated phenomenon. Although Benito might even have collaborated in altarpieces hitherto unanimously regarded as entirely autograph works by Juan de Flandes, the author believes that previous studies have linked Benito too exclusively to Juan's entourage. Indeed, the most tangible influence exerted on him seems to be that of Alonso Berruguete. In addition, there are strong indications of contacts with painters from the East of the Iberian Peninsula who had worked for some time in Renaissance Italy.
\end{abstract}

Punto de partida para cualquier estudio sobre el artista palentino ${ }^{1}$ Maestro Benito sería el bien conocido Martirio de las Once Mil Vírgines, mencionado como suyo en $1531^{2}$ y que adornaba entonces la capilla catedralicia dedicada a las santas (fig. 1). A partir de finales del s. XVIII, se encuentra colgado en la pared trasera de la Capilla Mayor, de espaldas a las tablas del Retablo Mayor, pintadas por Juan de Flandes, a quien suele considerarse como el maestro de Benito.

Sucio, pero en estado de conservación aceptable, el retablo, de forma casi cuadrada, está formado por cuatro anchos tablones que alcanzan una altura de casi tres metros. La línea del horizonte se halla muy alta, quedando sólo una cuarta parte de la altura del cuadro reservada para el cielo, que aún así se ve ampliamente tapado por vastos edificios con detalles renacentistas. Benito aprovecha esta distribución del espacio para mostrarnos casi una cincuentena de Vírgenes y otros tantos soldados, agrupados en forma serpenteada. La perspectiva carece de lógica, con ciertos descuidos alarmantes como en el caso de la extremada longitud del brazo

\footnotetext{
* Debo un profundo agradecimiento a Isabel Mateo Gómez y Rosario Serrano por sus comentarios y por haber corregido mi texto castellano.

1 Gracias a una relación de los vecinos de la parroquia de San Antolín, de 1533, se sabe que «Maestro Benito, pintor» vivió por entonces en la Calle de Santa Fé; ver Esteban García Chico, Palencia, Papeletas de Historia y Arte, Palencia 1954, p. 78.

2 En el 7 de agosto de aquel año dos canónigos están designados para averiguar cuanto se le debe a él y a un cierto Espinosa, por lo visto un escultor, por el retablo; según Alonso Fernández de Madrid, arcediácono de Alcor, el retablo se pagó con dineros cedidos por el arcediácono y canónigo D. Esteban de Villamartín, muerto en marzo 1525, lo que establecería un terminus post; ver Alonso Fernández de Madrid, Silva Palentina [1536-1539], edición de Matías Vielva Ramos, 3 tomos, Palencia 1932-1942, t. 2, p. 122 y nota 2.
} 
derecho del anciano a espaldas de Santa Úrsula. A su vez, la princesa coronada, que parece sostener una especie de diálogo último con el príncipe pagano, apenas se destacaría de sus compañeras si no fuese por el rico rojo de su manto purpúreo. Predomina el carácter de amalgama, y eso no sólo en cuánto a las formas sino, como veremos más adelante, también al estilo. Los defectos son aún más evidentes cuanto más se acerca uno al cuadro, quedando algo disimulados por el artificio de los efectos superficiales, cuando se ve de lejos.

A esta obra se sumaron en 1977 las cinco tablas pintadas, de poco más de un metro de ancho, que coronan un retablo en la parroquia de San Agustín del pueblo palentino de Capillas. Un Cristo Salvador en la gloria celestial, adorado por ángeles, está rodeado por sendas representaciones de los Apóstoles, de los Padres de la iglesia, de los Mártires y de las Santas vírgenes (fig. 2). Parecen restos del retablo que en 1536 aparecía citado en el antiguo hospital del lugar, dedicado efectivamente a Todos los Santos. Las tablas fueron publicadas por Jesús Urrea Fernández como «muy afín al Maestro Benito» ${ }^{3}$. Con motivo de su restauración en 1977-1978 en el ICROA (Instituto de Conservación y Restauración de Obras de Arte) madrileño, Juan Antonio Morán Cabré las describió un año más tarde, alegando cierta «pobreza compositiva», como una obra que Benito habría pintado antes del Martirio palentino ${ }^{4}$. Las ilustraciones publicadas por el Instituto confirman que los daños sufridos no impiden el análisis de las tablas y la formación de un juicio sobre ellas. Acertadamente se ha renunciado a una reconstrucción de las zonas perdidas que afectan sobre todo los extremos de los tableros individuales ${ }^{5}$.

Aparte de una Virgen actualmente de propiedad privada suiza ${ }^{6}$, cuadro de devoción de dimensiones y ambiciones mucho más reducidas, y que le fue atribuido a Benito, probablemente con acierto, en 1988 por Matías Díaz Padrón y Aída Padrón Mérida ${ }^{7}$, no se le ha adjudicado, según mis noticias, ninguna obra más.

Creo poder ampliar hoy, el catálogo de este importante pintor del foco palentino, con una tercera obra capital conservada en su primitivo lugar de destino, otra vez formando parte de la antigua diócesis de Palencia: el Retablo Mayor de la iglesia de San Pedro, en San Pedro de Montealegre, en la actual provincia de Valladolid. Este conjunto presenta el interés adicional de ser una obra de colaboración de dos artistas distintos, aunque sí parece que se hayan influído mutuamente. De Benito son sólo las seis tablas del lado del Evangelio, Oración en el Huerto y Prendimiento (fig. 3), Anunciación y Visitación, Liberación de San Pedro y el Quo vadis Domine (fig. 4). Las seis tablas del lado de Epístola que continuan la temática del Retablo con Descendimiento y Resurrección, Nacimiento y Adoración de los Magos así como con los Martirios de los Santos Pedro y Pablo, muestran una tendencia a composiciones más clasicistas, a la representación de figuras más pequeñas, a un colorido más claro y a un dibujo más suave y elegante (fig. 5).

\footnotetext{
3 Jesús Urrea Fernández, Antiguo Partido Judicial de Frechilla, en: Juan José Martín González (ed.), Ciudad de Palencia, Antiguos Partidos Judiciales de Palencia, Astudillo, Baltanás y Frechilla (Inventario artístico de Palencia y su provincia, t. 1), Madrid 1977, p. 119.

4 Juan Antonio Morán Cabré, «Instituto de Conservación y Restauración de Obras de Arte: Crónica de algunos trabajos realizados en el año 1978», en: Archivo Español de Arte, 51, 1978, pp. 357-364 y ill. 7-8: pp. 361/364.

María del Carmen Garrido [Pérez] (Sección de Pintura), en: Instituto de Conservación y Restauración de Obras de Arte, Catálogo de obras restauradas 1980-1982, Madrid 1984, ill. 113-122 (ill. 120 está al revés). En p. 87 otra vez como obra de Benito.

${ }^{6}$ Según pude averiguar, antes aparentemente en colecciones barcelonesas y neerlandesas así como en el comercio neoyorkino.

Matías Díaz Padrón y Aída Padrón Mérida, «Cuatro versiones de la "Virgen con Niño" por cuatro maestros castellanos del siglo XVI», en: Boletín del Seminario de Estudios de Arte y Arqueología (Valladolid), 54, 1988, pp. 394-402: pp. 396/399 e ill. 2. Mide cerca de 60 por $43 \mathrm{cms}$.
} 

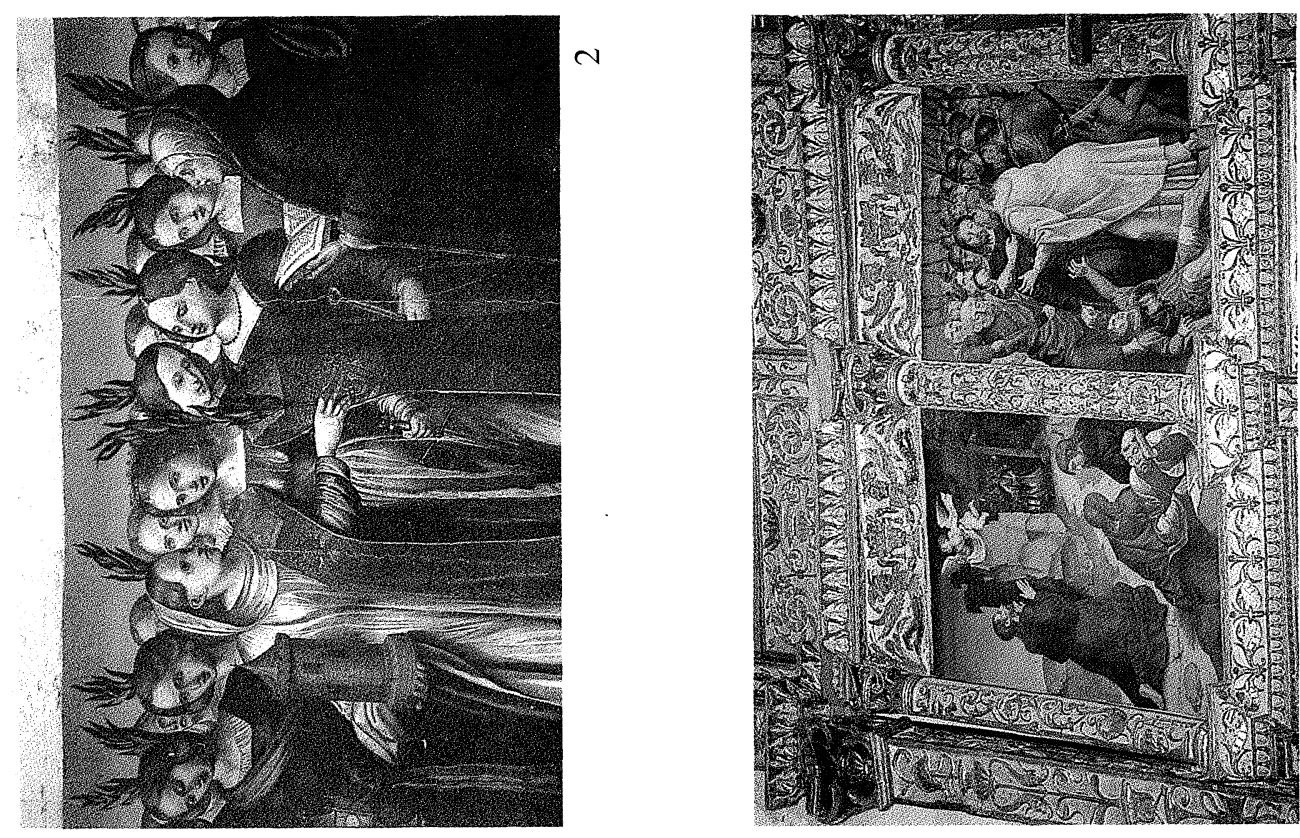

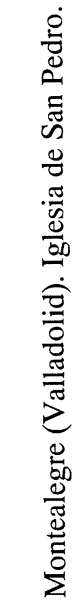
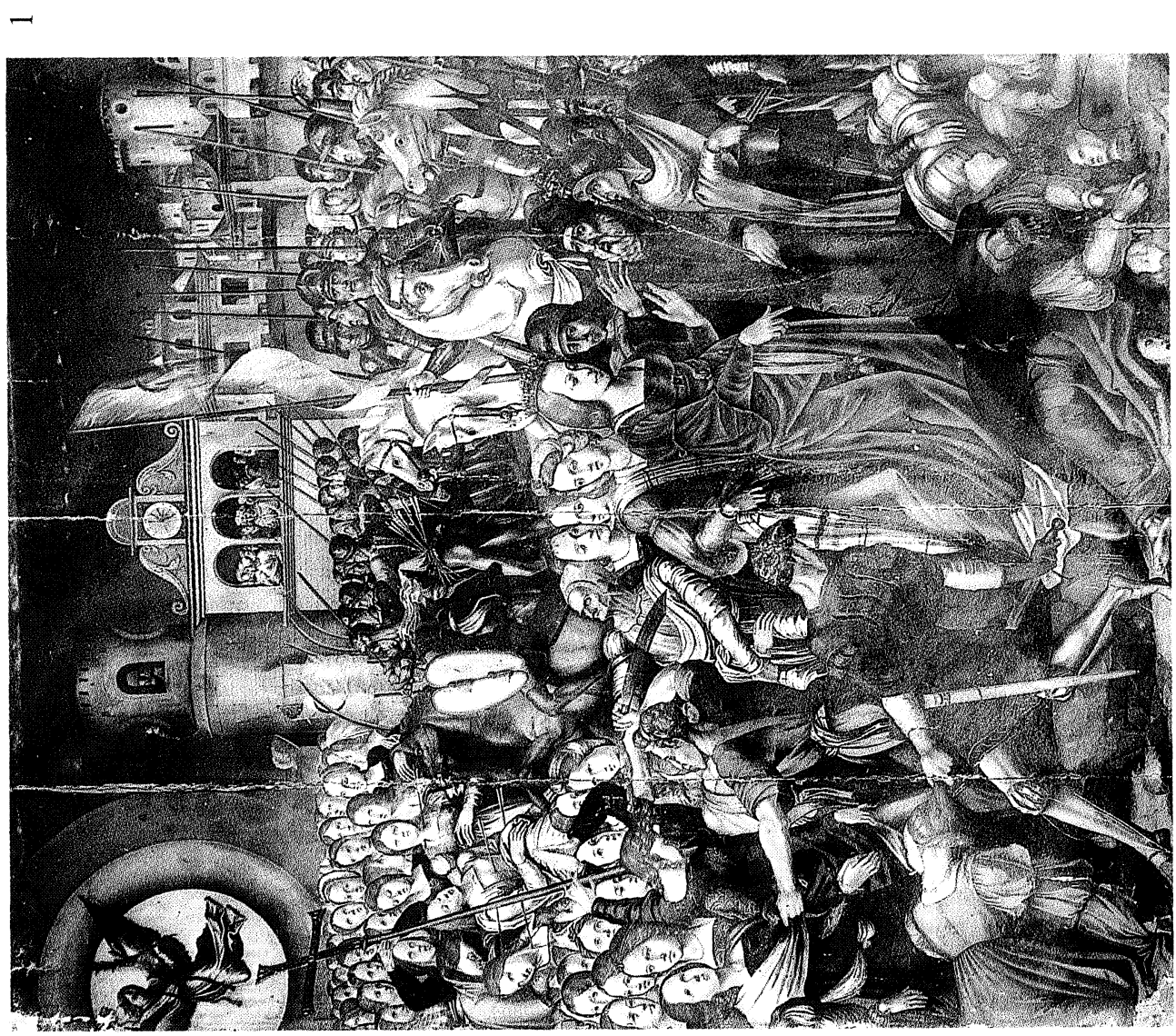

密早

荬

- 웅

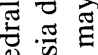

$\frac{\Phi}{5} \frac{0}{50}$ 음

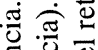

एँ

ป.

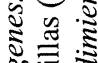

$=$ 난

$\approx 20$

원?

$\cong$ 인

डิ

8

$0, \overline{1}$

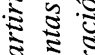

ปิ

.ํํㄹ.

颌

웅ㅇㅇ

$\sum \sum \sum$

$-i \dot{r}$

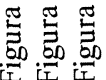


Cuando Esteban García Chico incluyó en 1959 tres fotos del retablo en el Catálogo Monumental de la Provincia de Valladolid, señaló que las pinturas podrían provenir del «foco palentino», tratándose quizás de obras de un buen colaborador del taller de Juan de Flandes ${ }^{8}$. Camón Aznar las describió en 1970 como de la «órbita» de este último ${ }^{9}$. Desconozco más referencias. La restauración llevada a cabo antes de $1991{ }^{10}$, aparentemente tampoco abrió un nuevo debate. Parece que las pinturas no se relacionaron jamás con Benito.

Sin embargo, son típicas obras suyas, desde las siluetas alargadas de los protagonistas al colorido. Otra vez se destacan algunas grandes y resplandecientes superficies rojas en un entorno predominantemente de tonalidades azuladas y grisáceas, enriquezido por acentos verdes, a su vez complementados por marrones y amarillos. El retablo muestra cabezas pequeñas, pero igualmente alargadas y con esta expresión tan característica del artista, con rasgos casi caricaturescos y una cierta predilección por las facciones vistas de perfil. Como en Palencia y Capillas, también observamos que manos y pies suelen ser excesivamente largos. Se repiten tanto las grandes superficies de algunos pliegues de Capillas, como las arquitecturas simplificadas y no muy bien dibujadas de Palencia. El Malco recuerda el gusto con que Benito pinta las figuras torcidas de los caídos en el martírio palentino. En el San Juan y otros Apóstoles de la misma escena, que en su desesperación dirigen cabezas y ojos al cielo, se repiten los gestos de desvanecimiento de algunas de las Vírgenes de Palencia. El angelito que presenta el cáliz al Cristo en Getsemani es hermano gemelo de aquellos del cuadro central de Capillas.

La permanencia del retablo en el lugar para el cual había sido destinado, ofrece la oportunidad de encontrar algun día más datos relativos al encargo. En el caso de una cuarta obra primordial de Benito, por el momento hay que renunciar a tal expectativa: A pesar de muchos esfuerzos, han fracasado todos mis intentos de saber más tanto sobre la procedencia como sobre el paradero actual de un Retablo de San Miguel que ha circulado por el mercado de París (fig. 5). Lo único que he podido averiguar con seguridad es que salió por primera vez a la luz en 1953, siendo objeto de una subasta en Drouot como «primitivo español» ${ }^{11}, \mathrm{y}$ que, después de haber pertenecido un tiempo a la casa de Brimo de Laroussilhe, desapareció nuevamente en otra subasta de Drouot, en 1987, entonces clasificado como de «escuela hispano-flamenca hacia $1540 »{ }^{12}$. Parece probable que saliera de España trás la llamada cruzada nacional, cuando los mismos párrocos fueron los que vendieron varios retablos palentinos

8 Esteban García Chico, Partido Judicial de Medina de Ríoseco (Catálogo Monumental de la Provincia de Valladolid, 2), Valladolid 1959, p. 48 y láms. 45-47.

9 José Camón Aznar, La pintura española del siglo XVI (Summa Artis, Historia General del Arte, t. 24), Madrid 1970, p. 128.

10 Fecha de una amable comunicación del sacérdote Luis Graña al autor. Aprovecho esta ocasión para expresar mi profundo agradecimiento a los vecinos responsables de la iglesia por la asistencia que me prestaron cuando estudié el retablo en 1997.

1 Primitifs espagnols, Sculptures en bois et en pierre du moyen âge et de la renaissance [...] provenant de la collection de Monsieur C..., dont la vente [...] aura lieu Hôtel Drouot [...], 27 Avril 1953, lote 1.

12 Objets d'art et de très bel ameublement [...], Dessins et tableaux anciens [...], Vente aux enchères publiques à Paris, Nouveau Drouot [...], 16 mars 1987 [...], 17 mars 1987 [...], p. 100 y reproducción color [que confirma lo que ya se vislumbra por el diseño], lote 139. Según el catálogo, el retablo medía cerca de 223 x $221 \mathrm{cms}$ (en 1953 se habían dado 230 x $225 \mathrm{cms}$ ). Por su asistencia en la obtención de estos datos, agradezco su ayuda a M. Dimitri Salmon del Departamento de Pinturas del Musée National du Louvre así como a M. Étienne Bertrand de la compañía Brimo de Laroussilhe. Todos los demás comerciantes implicados en la última venta no me sabían asistir a llegar más adelante. 
que hasta entonces habían permanecido intactos y en los templos para los cuales se habían hecho. Este ejemplar no ha sido nunca publicado ni atribuido a Benito ${ }^{13}$.

En este caso es todavía más lamentable que se desarraigara el retablo del lugar para el que fue concebido ya que la inscripción de la base del marco original nos da a conocer los donantes. Por desgracia, estos valiosos datos no están trascritos en los dos catálogos de venta con la precisión debida a pesar de que las fotos omitan los caracteres que se encuentran en los laterales de la base dentada. Según se observa en estas, el texto rezaría algo como: «Esta obra mando hazer Ivo Bianco ..deva Ramiel e Anner Garcia su muger e acabose en el año del señor de mil quenientos e XXXVI». Los nombres Bianco y Anner son poco probables y han sido completados aquí según la traducción al francés ofrecida por los catálogos. La fecha, sin embargo, parece segura y atestigua que este retablo es de algunos años posterior a los encargos de Palencia y Capillas.

Queda la remota esperanza de conocer a los donantes por otro camino y de identificar de esta manera el destino original del retablo. Su reducido tamaño y su temática hacen probable un contexto funerario. La iconografía está escogida con esmero poco habitual, implicando un encargo docto y ambicioso. Cada una de las seis escenas narrativas está protagonizada por un ángel. Aunque San Miguel haya sido relacionado con muchos sucesos distintos abarcando todo el período desde la Creación hasta el Juicio Final, no puede tratarse de una misma persona en todas las tablas. El San Miguel vencedor del diablo, imagen central del retablo, está flanqueado por la Anunciación y por la escena de los Ángeles sirviendo al Fruto de la Encarnación, después de que este hubo resistido a las tentaciones de aquel mismo diablo. En el fondo se ve a Cristo y Satanás encima del templo. A su vez, el fondo de la tabla central nos muestra la procesión al monte Gargano (?). En una especie de predela siguen la Curación de Tobías ${ }^{14}$, el Destierro del Paraíso por el ángel identificado tradicionalmente con San Miguel (que en la escena de arriba lucha contra el mismo Satanás que fue la causa de la perdición), y finalmente la Aparición a Bilaán, para los contemporáneos otro de los muchos papeles jugados por Miguel. Como remate de este programa bien pensado, se sitúa al Padre Eterno, rodeado por los coros de los ángeles, en el coronamiento del retablo, como punto de partida del Espíritu Santo que en forma de paloma y con su aureola correspondiente desciende sobre la Virgen. Aunque tales sugerencias exigen suma cautela, es posible que el grupo de observadores de la Curación de Tobías contenga alusiones a los donantes. Curiosamente, la mujer al lado de Tobías recuerda hasta cierto punto a aquella vestida al estilo de la época, que de manera parecida se incluye en la Visitación de Montealegre (fig. 4).

Artísticamente, Benito emplea una vez más las fórmulas que ya conocemos de las otras cuatro obras suyas. El angelito del Destierro se asemeja a aquel del cuadro de Getsemani, el perfil del ángel en la escena de Bilaán a los perfiles de Palencia y Capillas. En las piernas del ángel se intenta una articulazión de los músculos como la observamos en el Malco del Prendimiento. El plisado de su fino tejido o el del Gabriel de la Anunciación tienen un eco en algunas de las Vírgenes de Palencia así como en el Pedro y los Padres de la iglesia de Capillas. Los esbeltos óvalos, sobre todo de algunas caras femeninas, con los pequeños ojos demasiado pegados a la esbelta nariz, confieren a los rostros de Gabriel, de María o de Cristo la misma expresión extática y aquel aire de trance místico que ya conocemos de las Vírgenes

13 Sin embargo, D. Matías Díaz Padrón, conservador del Prado, me comunicó oralmente que había llegado a las mismas conclusiones, basándose como yo en las fotos reunidas por el Institut Amatller, de Barcelona. Algunas de las obras que cito a continuación han sido reclasificadas en el Institut Amatller bajo el nombre de Benito, supongo que a instancias del mencionado experto.

14 No reconocido en el catálogo de venta de 1987 («Agonie d'un Saint»). 
de Capillas. En relación con las obras anteriores, esta tendencia a crear un clima de exaltación mística parece haberse intensificado más todavía. Como antes, contribuyen a él las esbeltas proporciones, el movimiento agitado de los pliegues, la indicación altamente sumaria de arquitecturas y espacios. Aquí, sin embargo, Benito sitúa las grandes siluetas aún más en el primer plano, hacia el espectador. Los recortes son aún más audaces, los detalles que se desvanecen aún más numerosos, las figuras más incorpóreas, los espacios más irreales.

La serie de obras más o menos seguras de Benito la concluyen dos puertas de tríptico con la Anunciación, de apenas medio metro de altura, con representaciones de los Santos Roque y Andrés en los reversos (fig. 7). Muy típico es el fuerte acento rojo de los labios. Las tablas pertenecen, por lo menos desde $1929^{15}$, a la catedral de León. Si este paradero fuese el primitivo, serían, entre las obras con procedencia conocida, las únicas destinadas fuera de la antigua diócesis de Palencia. Sin embargo, debido a sus dimensiones, se trata de un objeto de fácil trasporte. No debe sorprender que la obra de Benito a la que más se parece es aquella que se le asemeja más por su tamaño: El Retablo de San Miguel (fig. 6). Como aquel, parece que no ha sido nunca reproducida.

En el caso de tres obras igualmente inéditas y cuyo paradero una vez más se ignora, las posibles diferencias de tamaño y la inaccesibilidad impiden por el momento un juicio responsable. Habrá que esperar hasta que un día salgan de sus escondrijos. Se trata en primer lugar de tres escenas de la vida de San Juan Bautista, Predicación, San Juan visitado en la prisión y Degollación, con altura similar a las tablas de Capillas. La primera de estas tablas se encontró por lo visto en 1978 en una colección barcelonesa, la última en 1969 (?) en otra palentina. Otro conjunto dedicado al mismo santo y que medía aproximadamente unos 240 por $145 \mathrm{cms}$, se encontraba también en propiedad barcelonesa. El conjunto mostraba en la banda superior: Visitación, Bautismo y el Bautista frente al Juez, en la mediana: Nacimiento, Epifanía y Degollación, y en el banco medias figuras de los Santos Pedro, Juan Evangelista y Bartolomé. Por la forma de los marcos originales y por las medidas es casi seguro que a este conjunto pertenecía también una tabla con los Apóstoles Pablo, Santiago y Andrés, subastada en 1976 en Ginebra como obra de Juan de Flandes, siguiendo un juicio de Gudiol ${ }^{16}$. El conjunto original debería haber sido, pues, considerablemente más grande.

Finalmente, en una Venganza de Herodias de antigua (?) propiedad palentina (fig. 8) los defectos de dibujo son mucho más acusados que en las obras a los cuales me referí anteriormente, quizás a causa de tratarse de una creación temprana ${ }^{17}$. Todas estas tres obras se parecen entre ellas por algunas características de dibujo que las separan un tanto de las pinturas analizadas primero. Un detalle obvio es la manera, muy particular, de representar nubes, para la cual no existen paralelos en las demás obras.

15 Fecha en que se les sacaron los negativos de la Junta de Museos. Estos laterales sí que están ahora registrados como debidos a Benito en el Arxiu Amatller, mientras que en los correspondientes ficheros del Arxiu Mas siguen clasificados como obras de Juan de Flandes.

${ }_{16}$ Très importante collection de tableaux espagnols du XIIIe au XVIIIe siècle [...], La vente publique aura lieu à l'Hôtel Richemond [...], le lundi 21 juin 1976 [...], pp. 20-21. Según datos del Institut Amatller, Barcelona (donde ya figura como posible obra de Benito), el cuadro se encontraba en 1964 en la colección Wallace Simonsen, de São Paolo.

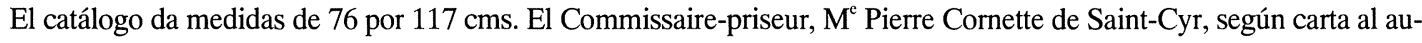
tor, en 1989 ya no disponía de datos que habrían podido servir para identificar el paradero actual de la obra.

17 Por los números de los negativos en el Amatller, no es de excluir que provenga del mismo retablo, como las tres escenas de la vida del Bautista. Todas las informaciones técnicas se basan en noticias del mismo Institut Amatller, donde el retablo de nueve escenas está ahora clasificado como debido a Benito, y las otras dos obras como procedentes del círculo o taller de Juan de Flandes. Según estas mismas fuentes, la Predicación de San Juan Bautista mide 114 por $75 \mathrm{cms}$. Las medidas para el segundo conjunto se refieren aparentemente al enmarcamiento original, antes de un posible desmontaje. 

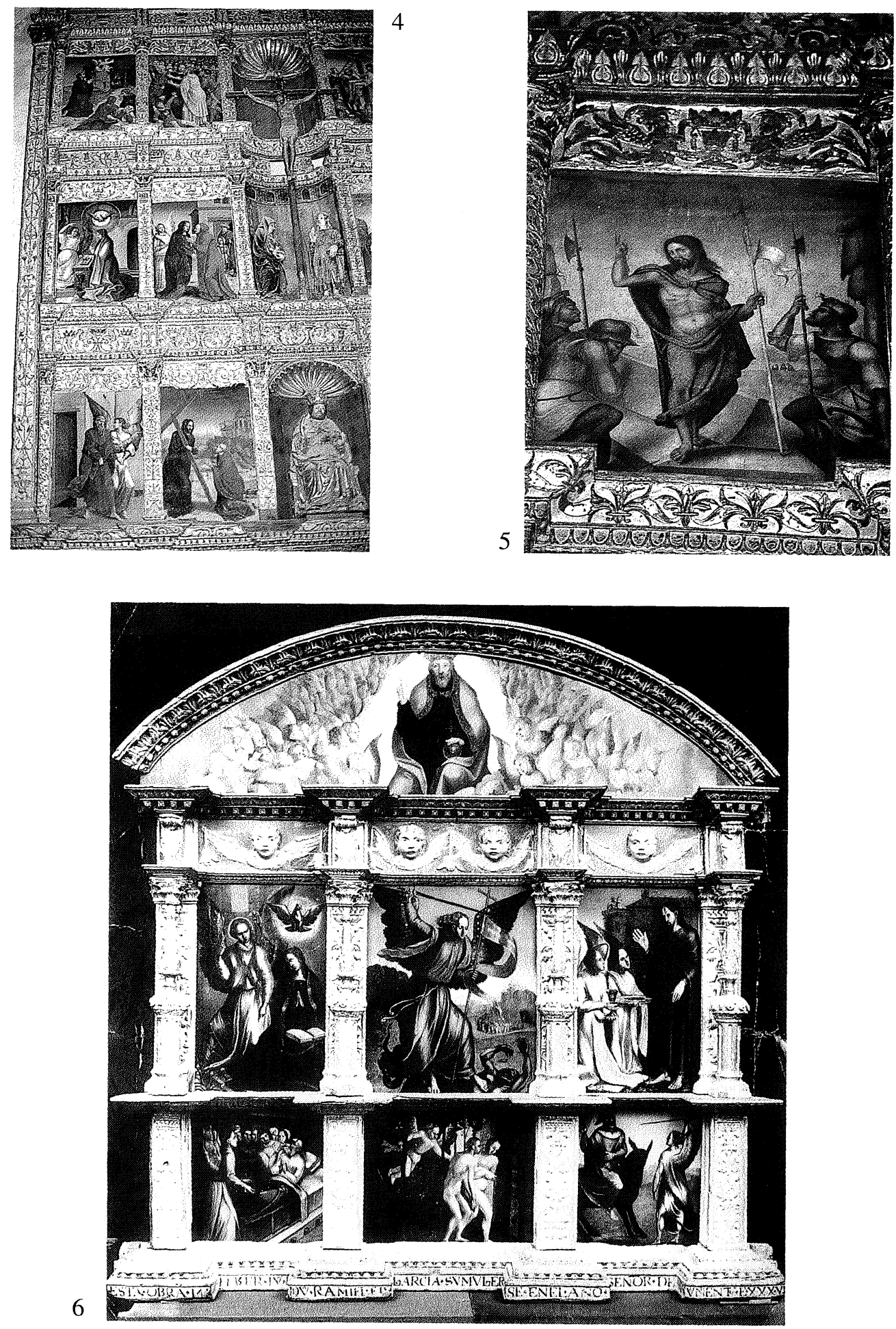

Figura 4. Maestro Benito. Escenas de la Pasión, Infancia y Vida de San Pedro. San Pedro de Montealegre (Valladolid). Iglesia de San Pedro.

Figura 5. Resurrección. San Pedro de Montealegre (Valladolid). Iglesia de San Pedro.

Figura 6. Maestro Benito. Retablo de San Miguel. Paradero actual desconocido. 

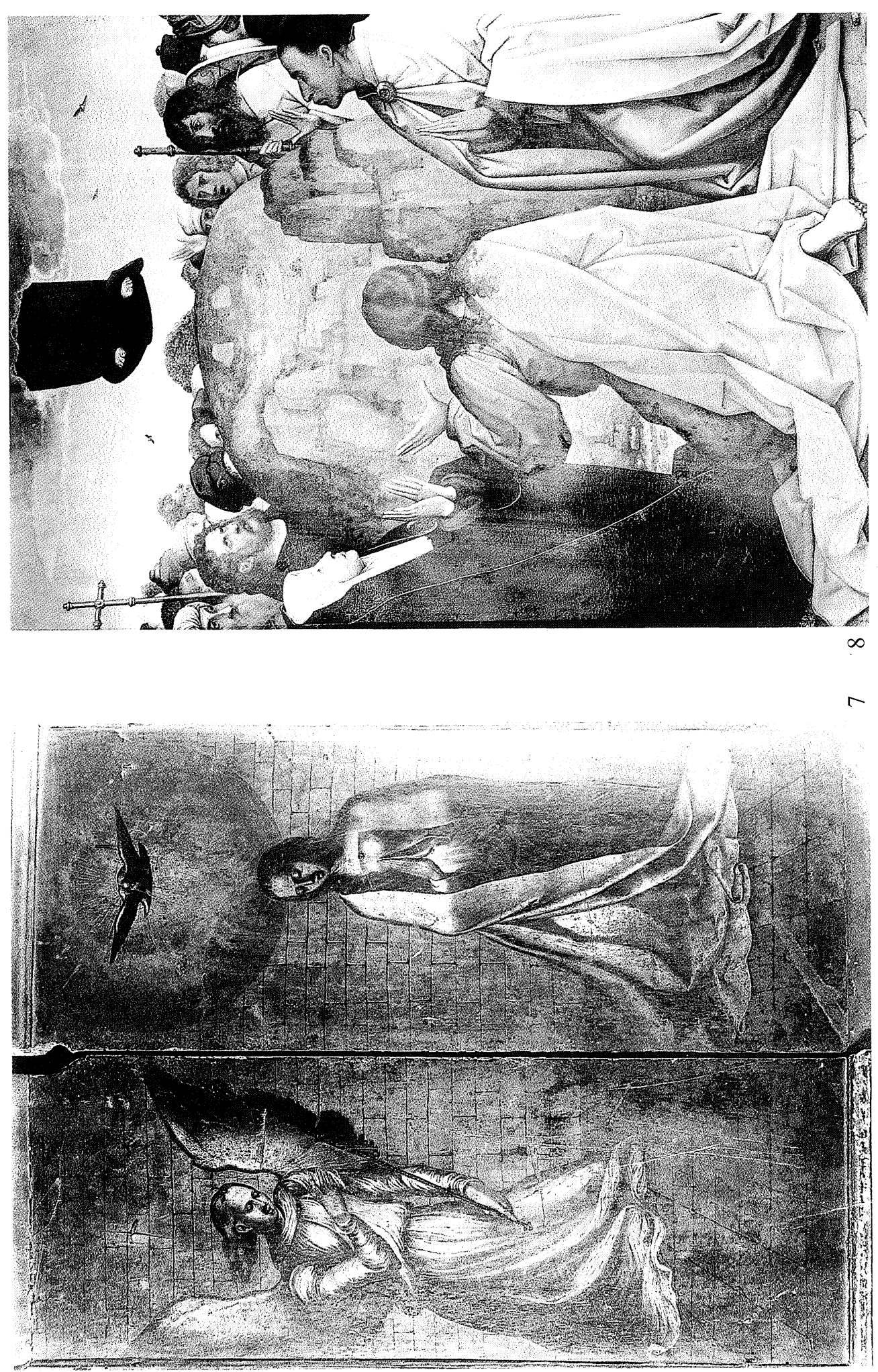

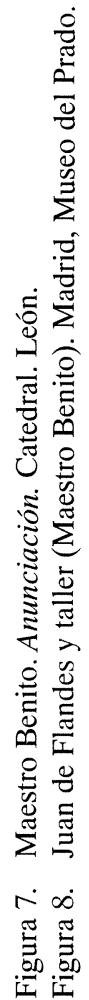


Por el modo de dibujar los ojos, estos tres conjuntos se asemejan a una Anunciación del museo de Raleigh que en los años sesenta había surgido en el comercio primero suizo y después neoyorkino como una obra de Juan de Flandes (fig. 9) ${ }^{18}$. Comparte las particularidades fisionómicas descritas para el núcleo de las obras de Benito y también presentes en el grupo de retablos del Bautista. Debido a las manipulaciones en el soporte, ya no se puede decidir si los recortes laterales se deben a las preferencias estéticas antes descritas o a circunstancias posteriores ${ }^{19}$. Si tuviesemos que distinguir dos manos distintas en este grupo de diez obras, la Anunciación de Raleigh quizás debería asignarse al pintor de los retablos del Bautista.

Sea cual fuese la actitud que uno quisiera tomar frente a estos cuadros, se concluye que un juicio definitivo sobre el total de la producción de Benito debe esperar hasta que exista un día la posibilidad de un estudio comparativo de todos los originales bajo condiciones similares, y más de cerca de lo que es normalmente posible en Palencia, Capillas y Montealegre. Sin embargo, la lista podría prolongarse casi infinitamente, con canditatos siempre un poco menos probables. Estos son prueba irrefutable de que el estilo de Benito no represente en absoluto un fenónemo aislado. Había varios artistas pintando de forma similar, y todos ellos se insertaban en unas tendencias generalizadas que se difundieron mucho más allá de la antigua diócesis de Palencia, por lo menos hasta zonas de Valladolid, Salamanca y otras tierras de Castilla la Vieja.

Esto nos lleva al segundo aspecto de estas consideraciones, al ambiente en el cual se había formado y desarrollado el estilo de Benito. Hasta ahora sólo se le ha considerado como un seguidor de Juan de Flandes, probablemente debido al hecho de que dos de las tres obras que se habían dado a conocer como suyas se encuentran en localidades palentinas y de que se le cita a Benito en relación con el Retablo Mayor de la Catedral de Palencia y las cuentas relativas a él, por lo menos estas no concluídas a la muerte de Juan de Flandes en 1519. El 14 de marzo de 1522 maestre Benito pintor veçino de Palençia y un tal Juan Texerina veçino de Paredes [de Nava], pintor reciben una tabla de oratorio de tress pieças con la cual el notario de la catedral, Alonso Paz, se había quedado como prenda. Como contrapartida, los dos artistas se comprometen a restitituir cualquier suma que se le habría podido pagar a Juan de Flandes en exceso a lo debido ${ }^{20}$.

Las obras mismas, sin embargo, facilitan menos indicios para tal relación de lo que autores anteriores han hecho sospechar. Se trata sobre todo de motivos como las aureolas estilizidadas alrededor de la paloma del Espíritu Santo o de algun personaje santo, las cuales Benito reproduce en Palencia, Montealegre, León y en el Retablo de San Miguel. También en Montealegre, el Cristo del Getsemani y las cabezas de los apóstoles Juan y Pedro, que le acompañaron al huerto de los olivos, copian bastante fielmente al cuadro correspondiente en el mencionado Retablo Mayor palentino. El San Juan repite todavía más literalmente la reprensentación del mismo santo en la Ascensión del retablo de la iglesia palentina de San Lázaro (hoy en el Prado; fig. 10), que, sin embargo, como veremos no me parece que se pueda atribuir enteramente a Juan de Flandes.

18 North Carolina Museum of Art, Acc. 65.9.1. La atribución a Juan de Flandes en Grosse Kunstauktion [...], Sehr bedeutende Gemälde alter und neuer Meister [...], Auktion in Luzern vom 13.-19. Juni 1961, Galerie Fischer [...], p. 109 y lám. 47, lote 1983, sigue un dictamen de Josep Gudiol Ricart. Sin embargo, ya Edward J. Sullivan, Catalogue of Spanish Paintings, North Carolina Museum of Art, Raleigh, North Carolina, Raleigh 1986, pp. 34-36, n.․․ 11, ha reconocido que la obra no sea de Juan mismo (ni de dos otros discípulos suyos que cita). El primer paradero conocido es, según él, la colección Ramón de Aras Juaregi, de San Sebastián. En el Amatller tampoco figura como obra de Benito.

19 Según una amable comunicación de David Goist, Chief Conservator, de abril 1989, la capa pictórica ha sido trasferida antes de la adquisición por el museo a un nuevo soporte. En ambos lados se añadieron sendas bandas de madera, los márgenes de la pintura original siendo irregulares.

20 Ver Jesús San Martín [Payo], El Retablo Mayor de la Catedral de Palencia, Nuevos datos, en: Publicaciones de la Institución «Tello Téllez de Meneses» 10, 1953, pp. 273-312: p. 294, y Ignace Vandevivere, $L a$ cathédrale de Palencia et l'église paroissiale de Cervera de Pisuerga (Les Primitifs flamands, I, Corpus de la Peinture des anciens Pays-Bas Méridionaux au quinzième siècle, t. 10), Bruselas 1967, pp. 70-71. 


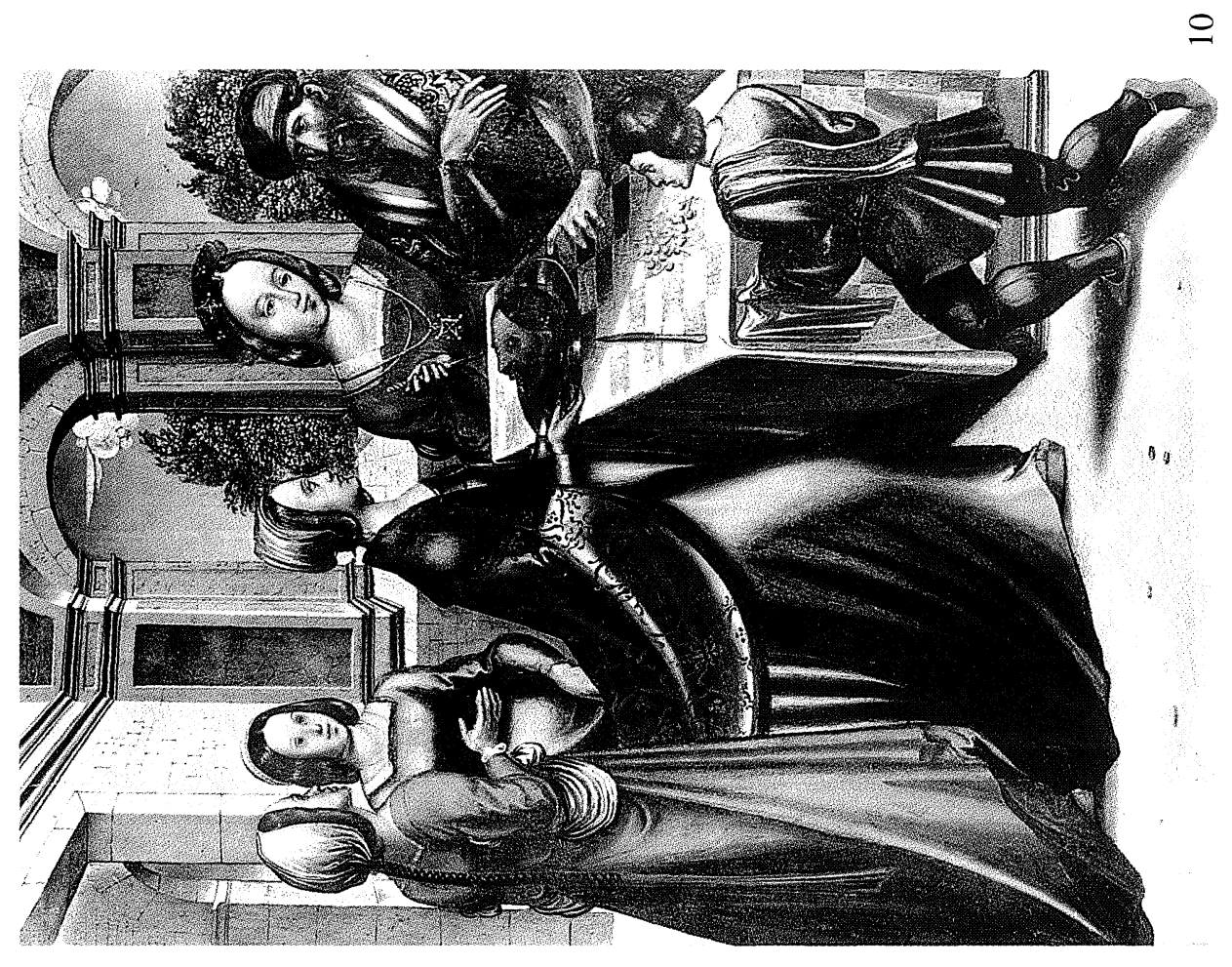

우 
Mesa y cerezas de la Venganza ofrecen una variación libre sobre la Cena de Emaús del retablo palentino. Juan de Flandes influye también en la paleta restringida de Benito y en la sobriedad de sus arquitecturas y escenarios. Benito le deberá a Juan incluso algunas de sus particularidades y habilidades técnicas como el sútil manejo del pincel y la aplicación jugosa de la pintura, haciendo suponer cierto uso de pigmentos ligados con óleo.

Incluso estaría dispuesto a dar un paso más audaz y proponer una colaboración directa de Benito en los dos retablos palentinos de Juan de Flandes. En la literatura sobre este último artista, desde las monografías de Elisa Bermejo de 1962 y, con Javier Portus, de 1988, hasta al grueso volumen dedicado por Ignace Vandevivere en 1967 al retablo catedralicio, desde los catálogos de exposición dirigidos por aquel mismo autor en 1985 y 1986 hasta a los catálogos del Prado y de la Galería Nacional de Washington, a los cuales museos pertenecen los restos del retablo de San Lázaro, los dos conjuntos se describen unáminamente como debidas en su integridad a Juan de Flandes ${ }^{21}$. Aunque ya parezca inverosímil por las confusiones en las cuentas para el Retablo Mayor, brevemente aludidas más arriba, esto es rotundamente desmentido por la evidencia visual, en particular observando las tablas de San Lázaro.

Gana más fuerza este argumento precisamente por el hecho de que me parece posible relacionar algunos de los cuadros de San Lázaro con ciertas carácteristicas que surgen entre la multitud de obras debidas a seguidores de Juan de Flandes ${ }^{22}$. Mientras haya más coincidencias entre estas tablas con una mano que sin firme fundamento suele identificarse con aquel Tejerina antes mencionado ${ }^{23} \mathrm{y}$ de la cual una gran parte de las obras permanece también sin publicar, algunos detalles de la ya aludida Ascensión en el Museo del Prado (fig. 8) apuntan a una posible intervención del propio Benito. La Virgen y el apóstol cuyos rostros flanquean la escena parecen ofrecer un preludio para los perfiles tan encarecidos por el artista. El apóstol imberbe detrás de la colina anuncia las expresiones extáticas del Prendimiento de Montealegre (fig. 7). En sus compañeros se advierte cierta semejanza con los apóstoles de Capillas. La larga diagonal de la orla bordada en oro que acentúa la figura de la Virgen tiene su contrapartida en las Marías de Raleigh (fig. 4) y en el Retablo de San Miguel (fig. 5). Teniendo en cuenta las preferencias estéticas de Benito, no parece mera coincidencia que en ninguna otra tabla del retablo de San Lázaro se exagera tanto la largura de las manos.

Prueba adicional de que Benito haya echado raíces dentro de la órbita de Juan de Flandes son las afinidades con el estilo de otros artistas del círculo de Juan y en particular con el

${ }^{21}$ Ver, entre otros, Elisa Bermejo [Martínez], Juan de Flandes (Serie Artes y artistas), Madrid 1962; Elisa Bermejo [Martínez] y Javier Portus, Juan de Flandes (Serie Los Genios de la Pintura Española, vol. 17), Madrid 1988; Vandevivere, La cathédrale de Palencia..., op.cit.; idem, Juan de Flandes, Europalia 85 España, Brugge, Memlingmuseum, Sint-Janshospitaal/Louvain-la-Neuve, Musée universitaire, Brügge 1985; idem (Preparacíon, texto y catálogo), Juan de Flandes, Museo del Prado, Febrero/Marzo 1986; John Oliver Hand and Martha Wolff, Early Netherlandish Painting (The Collections of the National Gallery of Art, Systematic Catalogue), Washington y Cambridge (MA) 1986 (ver en particular pp. 123, 125 y 130 131). La relación se podría prolongar infinitamente, también con publicaciones salidas en los últimos años. La única voz discrepante es la noción sumaria y no comentada en Colin [Tobias] Eisler, European Schools Excluding Italian (Paintings from the Samuel H. Kress Collection, t. [4]), Oxford 1977, p. 185, de que las tablas se deban a Juan «y colaboradores».

${ }^{22}$ Curiosamente, ya autores anteriores suelen hablar de una escuela de Juan de Flandes aunque insisten que éste no haya dirigido un equipo sino pintado todo de su mano, siguiendo al pie de la letra el contrato de 1509, cuyas otras cláusulas se sabe por cierto que no se respetaron; debierase asumir, pues, una enseñanza ejercida a través de visitas al taller o de clases nocturnas...

23 Ver en particular Matías Díaz Padrón, Una «Visitación» de Juan de Tejerina en el Museo del Prado, en: Boletín del Museo del Prado 6, 1985, pp. 134-137. 
del supuesto Tejerina, que yo preferiría llamar más neutralmente, basándome en una de las pocas obras suyas cuyo paradero actual se conoce, Maestro de Cincinnati ${ }^{24}$. Éste es el seguidor más fiel de Juan de Flandes, pero se trata de una lealtad trás de la cual se esconde una falta de fuerza creativa propia. No sorprende, pues, que también adopte fórmulas de Benito, artista más inventivo que él. Esta interrelación que se suma a la común dependencia de las fórmulas de Juan puede llegar hasta tal punto que amenace con obstruir la atribución correcta de algunas obras ${ }^{25}$. Comparte además las preferencias colorísticas de Benito.

A pesar de todo esto, las estrechas vinculaciones de éste con el círculo de Juan de Flandes no alcanzan a explicar los aspectos más llamativos de su arte, las proporciones alargadas de las figuras, los movimientos extáticos, «manieristas», el interés anatómico, la sutileza de las sombras, aquel «sfumato» italianizante; ni tampoco explican las fisionomías, tanto femeninas como masculinas. Creo que para todos estos fenónemos se puede aducir un sólo modelo, tratándose además de otro artista de primer rango y dentro de la misma diócesis de Palencia. La relación me parece tan evidente e íntima que no llego a explicarme como no ha sido evocado ya mucho antes ${ }^{26}$ : Me refiero a la relación de Benito con el arte de Alonso Berruguete.

Hijo de Pedro Berruguete, el escultor y pintor Alonso Berruguete era indiscutiblemente el artista castellano más importante de su generación. Poco antes de la muerte de Juan de Flandes, regresó de su década en Italia, aunque tardó todavía algunos años en volver a su nativa Castilla la Vieja ${ }^{27}$. Más allá de los tipos, sus pinturas comparten con aquellas de Benito la predilección por el plisado de las finas telas, por las crestas temblantes de los pliegues, los bordes titilantes de los tejidos, y por las grandes superficies lisas con las cuales éstos suelen a veces contrastar. Lo único que les falta a los vestidos de Benito es aquella impresión de estar esculpidas en piedra que Alonso sabe dar a los suyos. Aunque Alonso haya pintado el retablo del convento toledano de Santa Úrsula sólo una década más tarde, las posiciones algo inestables de varias figuras del banco del Retablo de San Miguel (fig. 5) parecen presuponer modelos berruguetianos.

No obstante, Alonso Berruguete no era la única vía por la cual Benito podía contemplar las inovaciones aportadas por Michelangelo y Leonardo. Como consta arriba, la parte derecha del retablo de Montealegre fue pintada por un artista completamente distinto a Benito. Esta incidencia es de un valor incalculable, considerando que la contribución de Benito al retablo es probablemente su obra menos típica y de que se trate por ende quizás de la primera manifestación de su arte. Ahora bien, la composición y el caracter general de la Resurreción (fig. 9) hacen recordar obras valencianas a la manera de los «Ferrandi», Llanos e Yáñez. Las semejanzas se extienden a tipos, colorido, modelado y hasta a la organización del paisaje. Esto adquiere máximo significado debido al hecho de que los valencianos se habían alimen-

24 Anunciación, Cincinnati Art Museum, n. 1958.88; Millard F. Rogers, Jr., Spanish Paintings in the Cincinnati Art Museum, Cincinnati 1978, pp. 11-12 y 37, como Juan de Flandes («hacia 1500»).

${ }_{25}$ Un caso ejemplar es una Virgen atribuida a Tejerina por Matías Díaz Padrón y Aída Padrón Mérida en el estudio citado en nota 8, p. 400-402. Es muy próxima a Benito por su dibujo, aunque menos por su ejecución.

${ }_{26}$ Única excepción - muy parcial - es la nota lúcida pero un tanto enigmática de Matías Díaz Padrón y Aída Padrón Mérida (op.cit., p. 396) de que Benito haya asumido («a diferencia de Tejerina») «la estética de Rafael y de los manieristas coetáneos».

${ }_{27}$ Para las pinturas de Alonso - que igualmente merecerían más atención y sobre todo un estudio de conjunto tanto de las obras pintadas en Italia como de aquellas realizadas a su vuelta a España - véase, entre muchas otras cosas, Emilio García Lozano, Catálogo de la exposición antológica de Alonso Berruguete en el $V$ centenario de su nacimiento (1491-1991), Palencia 1991, y últimamente Fernando Benito Doménech y Fiorella Sricchia Santoro (a cargo de), Ferrando Spagnuolo e altri maestri iberici nell'Italia di Leonardo e Michelangelo, Firenze, Casa Buonarroti, 19 maggio-30 luglio 1998, pp. 218-221 (con bibliografía actualizada). 
tado de las mismas fuentes que Alonso Berruguete. Así, antecedentes para los rostros de los dos soldados juveniles que se sobreponen de manera algo tosca los unos a los otros en el primer plano a la izquierda del martírio palentino (fig. 1) se hallan también e incluso más parecidos en obras de Pere Fernàndez, cuya estancia italiana se situaría por las mismas fechas que la de Alonso. En el Milagro de San Eusebio (?) de la predela del Retablo Mayor de Santa Maria delle Grazie a Caponapoli, de marcado carácter lombardo, se repite hasta el peinado con el pelo rizado, inspirado en esculturas clásicas y las clasicistas de Tullio Lombardo ${ }^{28}$. El mismo retablo de Nápoles ofrece prototipos sorprendentes -a su vez, inspirados en Leonardo- para la cabeza del verdugo anciano detrás de aquellos dos soldados de Palencia ${ }^{29}$. Sobre la frente de la serviente de la Visitación ${ }^{30}$ el tocado se alza en una especie de gran nudo, formando algo como una diadema, efecto al cual se alude en varias de las Vírgenes del martirio palentino. También esta idea se basa en esculturas clásicas. Ya antes de la descubierta del Apolón del Belvedere la podemos seguir en un artista como Francesco di Giorgio Martini ${ }^{31}$.

Varias son también las influcencias que se funden en la postura de los dos jóvenes soldados palentinos. Parecen salidos de algún sarcófago romano, tan del gusto de Alonso, y se asemejan más todavía a los ignudi que Michelangelo pintó en la bóveda de la Capilla Sixtina. El violento movimiento del primer soldado, con las piernas abiertas y el cuerpo doblado hacia atrás, parece como una variación Michelangelesca sobre el tema ofrecido por el San Juan del famoso Santo entierro de Rafael, de la Galería Borghese. Con todo esto, no es de extrañar que la oposición violenta entre una pierna echada hacia adelante y la otra hacia atrás vuelva a surgir también en el Milagro de San Eusebio, de Pere Fernàndez.

Benito ¿fue él mismo a Italia? Lo dudo, aunque dé a pensar que los rostros de dos de las Vírgenes en el extremo izquierdo del martírio Palentino (fig. 1), desviandose de los usuales estereotipos de Benito, se acercan más a modelos de Leonardo (y de seguidores suyos como Boltraffio o Ambrogio de'Predis) de lo que se suele observar en los demás españoles inspirados en aquel gran maestro. La obsesión de Benito por los perfiles es, al fin de cuentas, también una idea leonardesca, y hay cierto aire leonardesco en las propias formas. Sin embargo, y al contrario de lo que sucede con Pere Fernàndez, el Maestro del Retablo de

28 Nápoles, Museo e Gallerie Nazionali di Capodimonte. Ver ahora Marco Tanzi, Pedro Fernández da Murcia lo Pseudo Bramantino, Un pittore girovago nell'Italia del primo Cinquecento, Milán 1997, cat. 4, ill. p. 73, y el catálogo Ferrando Spagnuolo (nota 28), pp. 214-217, con referencias a la abundante literatura de los últimos años (que será pronto enriquezido por el párrafo dedicado a las «Riflessioni sull'esperienza milanese dello Pseudo-Bramantino» en A. Ballarin, Giovanni Antonio Boltraffio prima della pala Casio, de publicación imediata). En el círculo de los «Ferrandi», véase para cabezas parecidas a aquellos de los soldados de Benito una Degollación del Bautista en lugar desconocido, ill. ibidem, p. 188 (como Yáñez).

${ }_{29}$ Ver, entre otros, el pastor de la Adoración de los pastores, perteneciente a la duquesa de Villahermosa; Tanzi (nota 29), cat. 2. Figura también en Arte y cultura en torno a 1492, Exposición Universal Sevilla 1992, Sevilla 1992, p. 250 (texto de Joan Sureda i Pons). En qué medida Pere encarece tales tipos lo prueban los Santos José y el Zacarías de Visitación, Nacimiento y Adoración del mismo retablo de Santa Maria delle Grazie (otra vez en el museo de Nápoles; Tanzi, ill. p. 68-69, y Nicola Spinosa (a cargo de), Museo Nazionale di Capodimonte, Nápoles 1994, p. 42).

${ }^{30}$ Ver la nota anterior.

31 Ver, entre otras obras suyas, los dos Ángeles sosteniendo un candelabro de bronce, de la catedral de Siena, por lo visto fundidos en 1489-1490, y, todavía muy anteriores, los ángeles del fresco de la Virgen de la Villa Lodoli de Santa Regina, obra de colaboración con el llamado Fiducario di Francesco que fue trasladada a la Pinacoteca Nazionale de Siena y suele fecharse hacia 1470; figuran bajo los números 52 y 84 en el catálogo (editado por Luciano Bellosi) de la exposición monográfica Francesco di Giorgio e il Rinascimento a Siena 1450-1500, celebrada en Siena en 1993. 
Bolea, Paolo de San Leocadio o Alonso Berruguete, no parecen haberse conservado obras al Sur de los Alpes que apunten a su autoría. Además, fuera de aquel detalle el italianismo de Benito no llega nunca a ser tan profundo como él de los artistas mencionados. Invenciones de tanta osadía como las figuras aludidas de los dos jóvenes soldados en Palencia no vuelven a surgir en obras anteriores ni posteriores.

Mientras que las obras y la fama de Alonso Berruguete las tenía Benito a su vista en su propia patria palentina, sus contactos con las últimas corrientes valencianas están atestiguadas ya por la sóla estructura del retablo de Montealegre. Su caso ofrece, pues, un testimonio ejemplar de como en la figura de un destacado artista castellano de la tercera década del s. XVI se funden las tendencias venidas del Norte de Europa con las innovaciones llegadas desde Italia. 